cangrelor and compared this drug first with placebo and then with abciximab.

Cangrelor and abciximab proved equally effective in blocking platelet aggregation, but, because cangrelor has a shorter half-life, platelet activity returned to baseline levels more rapidly with this drug than with abciximab. Cangrelor was also associated with a trend toward shorter steady-state bleeding times than abciximab. Some patients who were given cangrelor experienced major bleeding events in the placebo-controlled part of the trial; a reduction in heparin dose and in the target activated clotting time greatly reduced the number of such events in the comparison with abciximab.

P2Y 12 -receptor antagonists block initial platelet activation by ADP, but do not affect the final pathway of platelet aggregation mediated by the fibrinogen receptor. It is believed that this mode of action of cangrelor, in combination with its short half-life, will allow inhibition of thrombosis during $\mathrm{PCl}$ without causing unwanted bleeding. In addition, heparin and aspirin in combination with $\mathrm{P}_{2} \mathrm{Y}_{12}$-receptor antagonists have a synergistic antithrombotic effect. The authors expect safety improvements in cangrelor therapy to arise from the elucidation of this relationship and less-aggressive heparin use.

Jim Casey

Original article Greenbaum $\mathrm{AB}$ et al. (2006) Initial experience with an intravenous $\mathrm{P}_{2} \mathrm{Y}_{12}$ platelet receptor antagonist in patients undergoing percutaneous coronary intervention: results from a 2-part, phase II, multicenter, randomized, placebo- and active-controlled trial. Am Heart J 151: 689.e1-689.e10

\section{Rotterdam Study confirms that many myocardial infarctions are clinically unrecognized}

Various studies have estimated that $4-44 \%$ of myocardial infarction (MI) events can remain unrecognized or 'silent' until patients' heart function is routinely assessed by imaging. As information is limited, however, de Torbal et al. sought to gather prospective data regarding the incidence of recognized and unrecognized $\mathrm{Ml}$ in relation to increasing age in a cohort of participants from the Rotterdam Study.

The Rotterdam Study included 7,983 men and women aged 55 years and older, of whom 5,148 individuals without evidence of prevalent MI were included in de Torbal et al.'s analysis.
The researchers compared the incidence of unrecognized and recognized Ml events according to age and gender; $\mathrm{Ml}$ events were considered unrecognized if they remained undetected from the baseline examination to the first or second follow-up.

After a median follow-up of 6.4 years, the incidence rate for $\mathrm{Ml}$ was nine events per 1,000 person-years, with, on average, four out of the nine events being classified as clinically unrecognized. Although the overall incidence of $\mathrm{Ml}$ events was lower among women than men, the proportion of unrecognized MI events was substantially higher among women (possibly because of the different ways in which the two sexes experience and report chest pain).

As a history of $\mathrm{Ml}$ is associated with an elevated risk of additional cardiovascular complications, the authors suggest that regular electrocardiography assessments would improve the detection and treatment of prevalent myocardial infarction in people aged 55 years and older.

Claire Braybrook

Original article de Torbal A et al. (2006) Incidence of recognized and unrecognized myocardial infarction in men and women aged 55 and older: the Rotterdam Study. Eur Heart J 27: 729-736

\section{Are patients with rheumatoid arthritis prone to accelerated atherosclerosis?}

Whether preclinical or early signs of atherosclerosis are more prevalent in patients with rheumatoid arthritis (RA) in unclear. In a crosssectional study, Roman and colleagues at the Hospital for Special Surgery, NY, sought to establish the prevalence of atherosclerosis in 98 consecutive outpatients with RA and 98 controls matched for age, sex and ethnicity.

Carotid ultrasonography was carried out in all patients, as were inflammatory marker assays and evaluation of cardiovascular risk factors such as hypertension, smoking and low serum levels of HDL cholesterol.

Compared with the controls, the prevalence of carotid atherosclerosis was three times higher in patients with RA, despite these patients having more-favorable risk factor profiles. Age, hypertension and therapy with tumor necrosis factor- $\alpha$ inhibitors were the only risk factors that correlated significantly with an increased likelihood of atherosclerotic plaque. 